\title{
Universality driven analytic structure of the QCD crossover: Radius of convergence in the baryon chemical potential
}

\author{
Swagato Mukherjee $\circledast^{1}$ and Vladimir Skokov $\circledast^{2,3}$ \\ ${ }^{1}$ Physics Department, Brookhaven National Laboratory, Upton, New York 11973, USA \\ ${ }^{2}$ Department of Physics, North Carolina State University, Raleigh, North Carolina 27695, USA \\ ${ }^{3}$ RIKEN/BNL Research Center, Brookhaven National Laboratory, Upton, New York 11973, USA
}

(Received 16 September 2019; accepted 19 March 2021; published 14 April 2021)

\begin{abstract}
Recent lattice QCD calculations strongly indicate that the chiral crossover of QCD at zero baryon chemical potential $\left(\mu_{B}\right)$ is a remnant of the second order chiral phase transition. Universal properties of this second order phase transition can be mapped to QCD temperature $T$ and $\mu_{B}$ using nonuniversal parameters determined by lattice QCD recently. Motivated by these results, first, we discuss the analytic structure of the partition function in the QCD crossover regime - the so-called Yang-Lee edge singularity—solely based on universal properties. Next, utilizing the lattice QCD results for nonuniversal parameters we map this singularity to the real $T$ and complex $\mu_{B}$ plane, leading to the determination of the radius of convergence in $\mu_{B}$ in the QCD crossover regime. These universality- and QCD-based results provide tight constraints on the range of validity of the lattice QCD calculations at $\mu_{B}>0$. Implication of this result on the location of the conjectured QCD critical point is discussed.
\end{abstract}

DOI: 10.1103/PhysRevD.103.L071501

\section{INTRODUCTION}

The chiral symmetry of quantum chromodynamics (QCD) is spontaneously broken in the vacuum. Firstprinciple lattice QCD calculations have conclusively shown that the approximate chiral symmetry with physical values of quark masses gets nearly restored at a pseudocritical temperature $T_{\mathrm{pc}}=156.5 \pm 1.5 \mathrm{MeV}$ [1] via a smooth crossover [2,3]. Lattice QCD calculations have also shown that similar chiral symmetry restoring crossover takes place at small-to-moderate values of $\mu_{B}$; the dependence of $T_{\mathrm{pc}}\left(\mu_{B}\right)$ has been computed [1]. It has been conjectured that at some sufficiently large values of $\mu_{B}$ the chiral restoration in QCD takes place through a first order transition; the point in the $T-\mu_{B}$ phase diagram at which the chiral crossover line turns into a first order phase transition line is known as the QCD critical endpoint (CEP), for a review, see Ref. [4].

While experimental searches to locate this conjectured QCD critical point are ongoing at RHIC and SPS, presently, first-principle lattice QCD calculations only provide very limited guidance on the existence and location of the QCD critical point in the $T-\mu_{B}$ phase diagram, because a direct

Published by the American Physical Society under the terms of the Creative Commons Attribution 4.0 International license. Further distribution of this work must maintain attribution to the author(s) and the published article's title, journal citation, and DOI. Funded by SCOAP ${ }^{3}$. lattice QCD calculation at $\mu_{B} \neq 0$ is hindered by the fermion sign problem. The present lattice calculations providing information on the QCD thermodynamicseither by carrying out Taylor expansions around $\mu_{B}=0$ [5] or through analytic continuation from purely imaginary values of $\mu_{B}$ [6-8]— crucially rely on the assumption that the QCD partition function is an analytic function of complex $\mu_{B}$ within a radius of convergence. To what extent these lattice QCD results are trustworthy, and how far in $\mu_{B}$ these methods might be extended can be answered only if we have reliable knowledge of the radius of convergence of the QCD partition function around $\mu_{B}=0$.

In this work, we extract this radius of convergence for the first time based on universal properties of QCD phase transition and first principal nonuniversal input from lattice QCD. To that end, we will provide an estimate for the location of the singularity nearest to $\mu_{B}=0$ and for $T \sim T_{\mathrm{pc}}$, based on the universal behavior of the QCD partition function with nearly massless $\mathrm{u} / \mathrm{d}$-quarks and mapping this universal structure to QCD $T$ and $\mu$ plane using nonuniversal input from (lattice) QCD calculations.

The universal analytic structure of the QCD crossover and its connection to the radius of convergence in complex$\mu_{B}$ plane were previously discussed in Refs. $[9,10]$, and a mean-field (random matrix) model-based estimate for the radius of convergence also was provided in Ref. [9].

The main idea is as follows. A system near a second order phase transition typically falls into one of the limited number of the universality classes. The universality class 
is fully defined by the global symmetries $(O(4)$ symmetry for the chiral phase transition in QCD) and the number of spatial dimensions $(d=3$ for QCD). The corresponding universal equation of state is a function of two variables: (i) The so-called reduced temperature, $t$, that does not explicitly break the symmetry of the system. The parameter $t$ measures the deviation from the second order phase transition point in the phase diagram. For finitetemperature QCD, $t$ is a combination of $T$ and $\mu_{B}$ (both parameters do not explicitly break $O(4)$ symmetry). (ii) The so-called magnetic field $h$-the relevant parameter that breaks the symmetry explicitly. In QCD, for the chiral phase transition, $h$ simply corresponds to the light quark mass. A true second order (chiral) phase transition takes place at $t=h=0$, while for any fixed $h \neq 0$ the system undergoes a smooth crossover transition as a function of the parameter $t$. This is manifest in the dependence of the equation of state on $(t, h)$ in the real domain (see, e.g., Ref. [11]). The apparent smoothness of the crossover equation of state disguises the singularity of the partition function at complex values of $(t, h)$ - the Yang-Lee edge singularity [12]. In the vicinity of a second order phase transition, in the scaling regime, and for a fixed value of $h$, this singularity limits the radius of convergence of a series expansions of thermodynamical observables in powers of $t$. Thus, the knowledge of the (universal) location of the Yang-Lee edge singularity and the (nonuniversal) mapping between $(t, h)$ and QCD variables $\left(T, \mu_{B}, m_{l}\right)$ would be sufficient in order to determine the radius of convergence. Recent theoretical advances in lattice QCD and Ref. [13] provide both required ingredients and allow us to extract the radius of convergence of the Taylor series of the pressure near zero baryon chemical potential.

\section{ANALYTIC STRUCTURE OF THE QCD FREE ENERGY FOR SMALL QUARK MASS}

Lattice QCD provides a compelling evidence that for massless u/d-quarks the chiral symmetry restoration in QCD takes place via a "true" second order chiral phase transition at $\mu_{B}=0$ (for a review, see Ref. [4]) [14]. Recent progresses in lattice QCD calculations have shown that for $\mu_{B}=0$ the chiral phase transition of $2+1$-flavor QCD, with massless up and down quarks and a physical strange quark, takes place at the chiral transition temperature $T_{c}^{0}=132_{-6}^{+3} \mathrm{MeV}$ [19]. The universality class of the chiral phase transition of $2+1$-flavor QCD was found to be consistent with that of the three-dimensional $O(4)$ spin model [19-22].

Based on the universal argument, if the light $\mathrm{u} / \mathrm{d}$-quark mass, $m_{l}$, is sufficiently small, the three dimensional QCD diagram of $m_{l}, \mu_{B}$, and $T$ can be mapped onto a onedimensional space using the so-called scaling variable, $z$. This mapping is suitable above the possible tricritical point. The behavior of the order parameter (the $\mathrm{u} / \mathrm{d}$-chiral condensate up to an overall constant) can then be described by [22]

$M\left(T, m_{l}, \mu_{B}\right)=\left(\frac{m_{l}}{m_{s}^{\text {phys }}}\right)^{\frac{1}{\delta}} f_{G}(z)+F_{\text {reg }}\left(T, m_{l}, \mu_{B}\right)$,

where the scaling variable, $z$, - a combination of three independent variables (light quark masses, $T$ and $\mu_{B}$ ) -is given by

$$
\begin{aligned}
z= & z_{0}\left(\frac{m_{l}}{m_{s}^{\text {phys }}}\right)^{-\frac{1}{\beta \delta}} \\
& \times\left[\frac{T-T_{c}^{0}}{T_{c}^{0}}+\kappa_{2}^{B}\left(\frac{\mu_{B}}{T_{c}^{0}}\right)^{2}+\kappa_{4}^{B}\left(\frac{\mu_{B}}{T_{c}^{0}}\right)^{4}+\cdots\right] .
\end{aligned}
$$

The form of the scaling variable follows from the one of the $O(N)$ spin model $z=z_{0} h^{-\frac{1}{\beta \delta}} t$. Here $h$ is the symmetry breaking field which in QCD corresponds to the light quark mass; while $t$ is the field responsible for the deviation from the criticality without explicit symmetry breaking; in QCD, a combination of $T-T_{c}^{0}$ and $\mu_{B}$ play the role of $t$. The chemical potential due to charge conjugation symmetry can contribute through even powers only.

Thus the analytic properties of $f_{G}(z)$ encode the entirety of the critical behavior in the scaling regime of $O(4)$ phase transition. That is the whole three dimensional QCD diagram in Fig. 1(b) for $T$ about the tricritical temperature with the analytical structure in the complex chemical potential plane in Fig. 1(c) and Fig. 1(d) can be read off from Fig. 1(a) and the properties of a single-variable function $f_{G}(z)$.

In Eq. (1), $f_{G}$ is the universal scaling function [23], $\beta$ and $\delta$ are the universal critical exponents; while $z_{0}$ and the curvature parameters of $T_{\mathrm{pc}}\left(\mu_{B}\right), \kappa_{2,4}^{B}$, are nonuniversal. Current lattice QCD calculations established that $\kappa_{4}^{B}$ is consistent with zero within the precision of the calculation [1]; based on this we set $\kappa_{4}^{B}$ and possible higher order corrections denoted by ellipses to zero in what follows. The physical strange quark mass is denoted by $m_{s}^{\text {phys }}$. For simplicity, we only consider the leading singular contribution in Eq. (1). There are also subleading scaling corrections (see, e.g., Ref. [24]). However, they do not modify the location of the singularities in $\left\{T, \mu_{B}, m\right\}$-plane and, therefore, are not important in the context of our work. The function $F_{\text {reg }}$ characterizes small deviations, if any, from the scaling behavior. Lattice calculations have provided evidence that chiral observables of QCD with physical values of $\mathrm{u} / \mathrm{d}$-quark masses are well described by Eq. (1) by including only small corrections from $F_{\text {reg }}[19,21,22,25]$. Since $F_{\text {reg }}$ is not expected to have any singularities close to zero chemical potential [26], the analyticity of $M$ in the complex $-\mu_{B}$ plane is governed by the analytic structure of the universal function $f_{G}$. The corresponding universal 

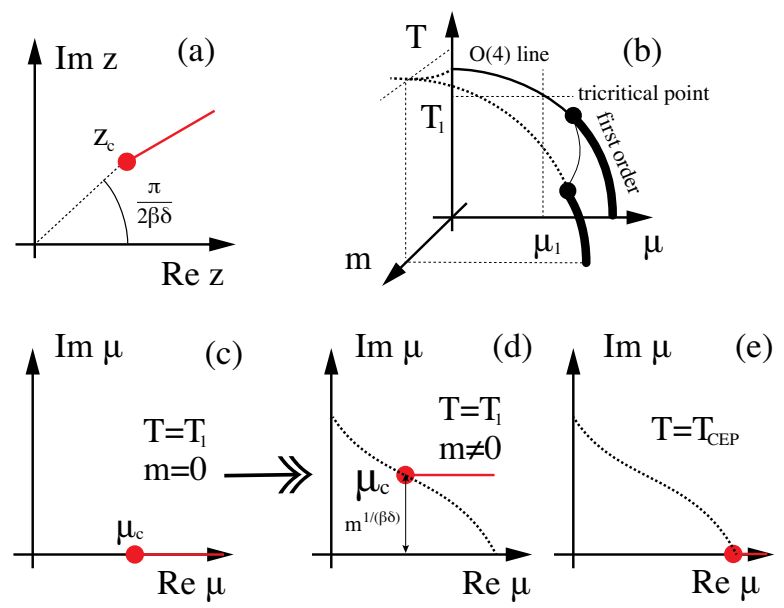

FIG. 1. Illustration of analytic structure of the universal equation of state and its mapping to QCD: (a) Yang-Lee edge singularity of the magnetic equation of state; the argument of the singularity is defined by the $O(4)$ critical exponents; (b) illustration of phase diagram in the plane of temperature-baryon chemical potential-light quark mass; note that all three variables can be combined into a single variable $z$ in the scaling regime, see Eq. (2); for the purpose of illustration, we consider an arbitrary temperature $T_{1}$ above the tricritical temperature in the chiral limit and not smaller than the temperature of the phase transition at zero chemical potential; (c) analytic structure in the complex chemical potential plane in the chiral limit for a fixed temperature $T_{1}>T_{\text {tricritical }}$; (d) analytic structure for nonzero light quark mass for $T_{1}>T_{\mathrm{CEP}}$ : the singularity from the real axis is shifted to the complex values of the chemical potential; this situation corresponds to the crossover phase transition; (e) the same as (d) but for $T=T_{\mathrm{CEP}}$ : the singularity and its complex conjugate (not shown) approach and pinch real axis resulting in a critical end point (CEP). $O(4)$ magnetic equation of state with mapping Eq. (2) fairly describes analytical structure in the $O(4)$ scaling regime.

chiral behavior of the QCD free energy is given by $\left(m_{l} / m_{s}^{\text {phys }}\right)^{1+1 / \delta} f(z)$, where the scaling function $f(z)$ is related to $f_{G}$ through: $f_{G}=z f^{\prime}(z) /(\beta \delta)-(1+1 / \delta) f(z)$; the prime denotes the derivative with respect to $z$.

It is well known that in the complex- $z$ plane the function $f_{G}$ has a singularity; it is the so-called Yang-Lee edge singularity [27] of the form $f_{G} \sim\left(z-z_{c}\right)^{\sigma}$ [12], see Fig. 1(a). The corresponding most singular contribution to the free energy is then $f(z) \sim\left(z-z_{c}\right)^{1+\sigma}$. Since there has been some confusion in the QCD literature, we comment that the critical exponent for this singularity of the free energy, $1+\sigma$ is not related to the $O(N)$ specific heat critical exponent, $2-\alpha$, as one naively might assume. Additionally, the critical exponent $\phi$ introduced in Ref. [10] for the crossover singularities is nothing but $\sigma$. The YangLee edge singularity can be treated as an ordinary critical point, belonging to the $Z(2)$ universality class of $\phi^{3}$ theory in three spatial dimension, and with a purely imaginary coupling [12]. Although, as long as it is positive, the exact value of $\sigma$ is not important for our analysis, we want to point out that it has been known for more than four decades - for all finite values of $N$ of three-dimensional $O(N)$ universality class $\sigma=0.085(1)[12,28]$. The argument of $z_{c}$ is known in terms of the $O(N)$ critical exponents [29] as a consequence of the Lee-Yang theorem $z_{c}=$ $\left|z_{c}\right| e^{i \frac{\pi}{2 \beta \delta}}$ as illustrated in Fig. 1(a). In contrast to the exponent and the argument of $z_{c}$, the absolute value $\left|z_{c}\right|$ was not know until recently, see Ref. [13]. We stress that owing to the universality of $f_{G}$ as a function of (complex) $z$ the value of $\left|z_{c}\right|$ is also universal. Analytically, $z_{c}$ can be calculated in two limits-mean-field and $N \rightarrow \infty$.

\section{ANALYTIC STRUCTURE OF $\boldsymbol{f}_{G}$ IN THE MEAN-FIELD AND $N \rightarrow \infty$ LIMITS}

In both limits, the mean-field and the $N \rightarrow \infty, f_{G}$ can be represented in the following general form [30]

$$
f_{G}\left[z+f_{G}^{2}\right]^{\gamma}=1 .
$$

The specific cases can be obtained by plugging in the corresponding critical exponents- $\gamma=1$ for mean-field, and $\gamma=2$ for $N \rightarrow \infty$. For the mean-field case this equation can also be obtained straightforwardly by varying the free energy of the $\phi^{4}$ Landau-Ginzburg theory [31] with respect to the order parameter $\phi$. To determine the Yang-Lee edge singularity branch-point, $z_{c}$, from $f_{G}$ we consider the inverse function $z\left(f_{G}\right)$. The branch-point can be obtained from the condition $\left(d z / d f_{G}\right)_{z_{c}}=0$. Considering only the branch closest to $z=0$ on the physical Riemann sheet, i.e., the one connected to $z=0$ as defined by the standard normalization condition $f_{G}(0)=1$, Eq. (3) and $\left(d z / d f_{G}\right)_{z_{c}}=0$ completely determine

$$
\begin{aligned}
z_{c}^{\mathrm{MF}} & =\frac{3}{2^{2 / 3}} e^{i \frac{\pi}{3}} & \text { mean-field }, \\
z_{c}^{N \rightarrow \infty} & =\frac{5}{2^{8 / 5}} e^{i \frac{\pi}{5}} & N \rightarrow \infty .
\end{aligned}
$$

\section{RADIUS OF CONVERGENCE IN THE COMPLEX- $\mu_{B}$ PLANE}

As evident from Eqs. (1) and (2), for a fixed value of $m_{l}>0$, the derivatives of $M$ with respect to $\mu_{B}$ are proportional to the derivatives $f_{G}(z)$ with respect to $z$. Thus, the convergence of the Taylor expansion in $\mu_{B}$ around $\mu_{B}=0$, as well as analytic continuation in the complex $-\mu_{B}$ plane are bounded by the value of $z_{c}$. Specifically, from Eq. (2) for a definite value of $T$ the Taylor series about zero chemical potential will have the radius of convergence given by

$$
R_{\text {conv }}=\left|\frac{z_{c}}{z_{0}}\left(\frac{m_{l}}{m_{s}^{\text {phys }}}\right)^{1 / \beta \delta}-\frac{T-T_{c}^{0}}{T_{c}^{0}}\right|^{1 / 2} \frac{T}{\sqrt{\kappa_{2}^{B}}} .
$$




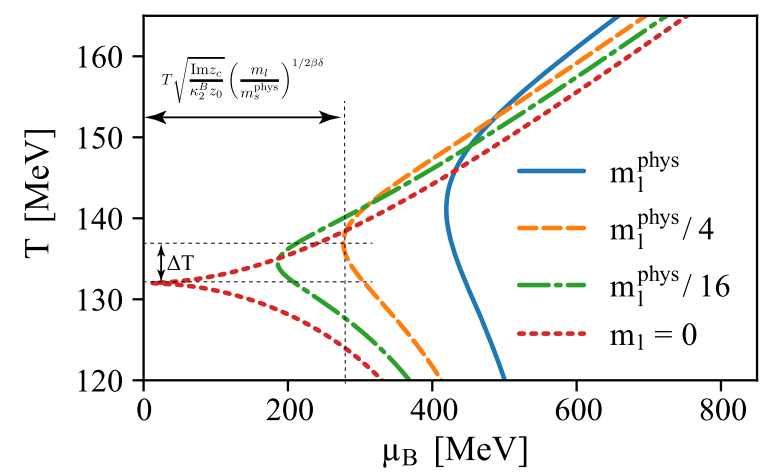

FIG. 2. Radius of convergence $\mu_{B}$ for different values of $T$ and for different values of the light up/down quark masses. The minimum of the curves shifts to higher temperatures by the amount $\frac{\Delta T}{T_{c}^{0}}=\frac{\operatorname{Re} z_{c}}{z_{0}}\left(\frac{m_{l}}{m_{s}^{\text {pys }}}\right)^{\frac{1}{\beta \delta}}$. See text for details.

At $T=T_{c}^{0}$, the radius of convergence is directly proportional to $\left|z_{c}\right|$. In general, $R_{\text {conv }}$ depends not only on the magnitude of $z_{c}$ but also on its phase. From this equation it follows, that the radius of convergence assumes its minimal value $T \sqrt{\frac{\operatorname{Im} z_{c}}{\kappa_{2}^{B} z_{0}}}\left(\frac{m_{l}}{m_{s}^{\text {phys }}}\right)^{1 / 2 \beta \delta}$ when the real part of the first term cancels completely the second term in Eq. (5). This minimum shifts to temperatures higher then $T_{c}^{0}$ by the amount $\frac{\Delta T}{T_{c}^{0}}=\frac{\operatorname{Re} z_{c}}{z_{0}}\left(\frac{m_{l}}{m_{s}^{\text {phys }}} \frac{1}{\beta \delta}\right.$. As expected both values are defined by $z_{c}$, see also Fig. 2 .

The previous discussion clearly demonstrates that, in the scaling regime, validity of the Taylor expansions in $\mu_{B}$ and the analytic continuations in complex- $\mu_{B}$ plane of the QCD free energy is determined by the value of $\left|z_{c}\right| .2+1$-flavor lattice QCD calculations show that the chiral condensate, $M$, for the physical value of light up/down quark mass, $m_{l}^{\text {phys }}=m_{s}^{\text {phys }} / 27$, are well described by the 3 -dimensional $O(4)$ scaling function $f_{G}$, with inclusion of small corrections from the analytic function $F_{\text {reg }}[19,21,22,25]$. Obviously, $F_{\text {reg }}$ unavoidably affects the values of the low-order Taylor coefficients; however, any analytic contribution does not change the radius of convergence. Base on these arguments, we expect that, for QCD, the singularity nearest to $\mu_{B}=0$ in the complex- $\mu_{B}$ plane is defined by $z_{c}$. If $z_{c}$ is known then Eq. (2) can be used to translate this singularity to the complex- $\mu_{B}$ plane and, thereby, determine the corresponding radius of convergence. The rest of the universal and non-universal parameters entering Eq. (2) are known-(i) The critical exponents of the $O(4)$ universality class $\beta=0.380, \delta=4.824$ [32]. (ii) Both $m_{l}^{\text {phys }}$ and $T$ are purely real. (iii) $T_{c}^{0}=132_{-6}^{+3} \mathrm{MeV}$ [19]. (iv) The curvature of the pseudocritical temperature $T_{\mathrm{pc}}\left(\mu_{B}\right), \kappa_{2}^{B}=0.012(2)$ [1]. (v) The scale factor $z_{0}$ can be determined by fitting the $m_{l}$-dependence of the lattice QCD-calculated $T_{\mathrm{pc}}\left(m_{l}\right)$ [3]; based on the lattice QCD results of Ref. [19] on $T_{\mathrm{pc}}\left(m_{l}\right)$ the scale factor is estimated to be $z_{0} \simeq 1-2$ [33]. Currently the best estimate for $\left|z_{c}\right|$ is

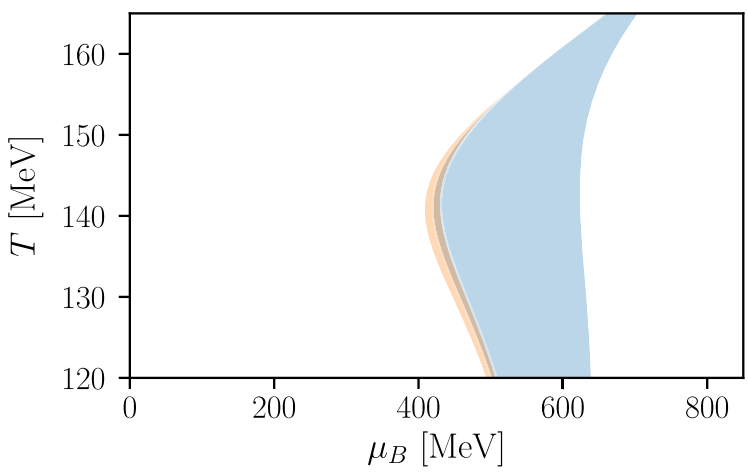

FIG. 3. Radius of convergence in $\mu_{B}$ for physical quark masses. The orange band is for $z_{0}=2$ and incorporates a $5 \%$ uncertainty on the value of $\left|z_{c}\right|$. The blue band depicts variation of $z_{0}=1-2$.

available from the functional renormalization group studies [13]; they show that $\left|z_{c}\right| \approx 1.665$ for $O(4)$. This value is accidentally close to the one obtained in the large $N$ limit $\approx 1.649$. In our analysis we use $\left|z_{c}\right|=1.665$. We note that the functional renormalization group approach is well suited for extracting the location of the edge singularity, as it does not rely on the Monte-Carlo importance sampling and thus does not suffer from the sign problem which hinders lattice simulation at complex (imaginary) values of $z(h)$. Moreover, critical behavior is dominated by the longrange physics of the slow critical modes. This justifies the derivative expansion of the effective action; in the nonperturbative functional renormalization group approach, this expansion is know to rapidly converge [35]. To account for a possible systematic uncertainty of the truncation scheme used in the functional renormalization group calculation we generously vary $\left|z_{c}\right|$ by $5 \%$. The large- $N$ value of $\left|z_{c}\right|$ falls into this uncertainty band.

In Fig. 2 we show the radius of convergence in $\mu_{B}$ in the $T-\mu_{B}$ plane for different values of $m_{l}$ in the range $0-m_{l}^{\text {phys }}$, using $z_{0}=2, O(4)$ critical exponents, and other lattice QCD-determined nonuniversal parameters described above. Note that, in the chiral limit, QCD free energy is singular at $T=T_{c}^{0}, \mu_{B}=0$ and, therefore, the radius of convergence at this point is zero, see also Refs. $[9,10]$.

Figure 3 provides a more realistic estimate for the radius of convergence in $\mu_{B}$ in the $T-\mu_{B}$ plane for $m_{l}^{\text {phys }}$ by varying $\left|z_{c}\right|$ around its FRG value and $z_{0}=1-2$. While the value of $\left|z_{c}\right|$ was recently determined to rather high precision and leads to a limited uncertainty of the radius of convergence, more precise lattice QCD result for $z_{0}$ is needed to improve this estimate.

\section{CONCLUSIONS}

Relying only on the universal behavior of QCD in the chiral crossover region we investigated the analytic behavior of the free energy. We argued that if the chiral behavior of QCD is well-described by the universal scaling, as borne 
out in recent the lattice QCD calculations, then the analytic structure of the free energy will be completely governed by the corresponding universal scaling function. We estimated the relevant singularity of the scaling function based on the two extreme limits of mean-field and $N \rightarrow \infty$. For the analysis, we used the location of the singularity extracted in functional renormalization group calculations. We showed how this can be translated to the singularity in the complex$\mu_{B}$ plane to determine the radius of convergence in $\mu_{B}$. Our results are solely based on the universal input and welldetermined nonuniversal parameters from lattice QCD calculations. Figure 3 summarizes our universality- and QCD-based estimate for the radius of convergence in $\mu_{B}$ for temperatures in the vicinity of the QCD chiral crossover. It shows that the radius of convergence is larger than $\left|\mu_{B}\right| \gtrsim 400 \mathrm{MeV}$, implying that the present lattice QCD calculations based on Taylor expansions in $\mu_{B}$ and analytic continuations from imaginary values of $\mu_{B}$ can be reliable below this region, as suggested also by recent lattice QCD calculations $[1,5,36]$.

The current state-of-the-art lattice QCD calculations do not find any evidence for an additional singularity for
$\mu_{B} \lesssim 400 \mathrm{MeV}[1,5,36]$. Our result on the radius of convergence $\left|\mu_{B}\right| \gtrsim 400 \mathrm{MeV}$, coupled with these lattice QCD results, suggest that QCD critical point, if one exists, will most likely be located at $\mu_{B} \gtrsim 400 \mathrm{MeV}$.

\section{ACKNOWLEDGMENTS}

This material is based upon work supported by the U.S. Department of Energy, Office of Science, Office of Nuclear Physics: (i) Through the Contract No. DE-SC0012704; (ii) Through the contract No. DE-SC0020081; (iii) Within the framework of the Beam Energy Scan Theory (BEST) Topical Collaboration. V.S. also thanks the ExtreMe Matter Institute EMMI (GSI Helmholtzzentrum für Schwerionenforschung, Darmstadt, Germany) for partial support and their hospitality. We thank Bengt Friman, Frithjof Karsch, Lex Kemper, Misha Stephanov, Robert Pisarski, Krzysztof Redlich, Thomas Schäfer, and Mithat Ünsal for illuminating discussions. We thank the organizers of EMMI Workshop "Probing the Phase Structure of Strongly Interacting Matter: Theory and Experiment", which inspired us to work together on this project.
[1] A. Bazavov et al. (HotQCD Collaboration), Phys. Lett. B 795, 15 (2019).

[2] T. Bhattacharya et al., Phys. Rev. Lett. 113, 082001 (2014).

[3] A. Bazavov et al., Phys. Rev. D 85, 054503 (2012).

[4] K. Fukushima and T. Hatsuda, Rep. Prog. Phys. 74, 014001 (2011).

[5] A. Bazavov et al., Phys. Rev. D 95, 054504 (2017).

[6] C. Bonati, M. D'Elia, M. Mariti, M. Mesiti, F. Negro, and F. Sanfilippo, Phys. Rev. D 92, 054503 (2015).

[7] R. Bellwied, S. Borsanyi, Z. Fodor, J. Guenther, S. D. Katz, C. Ratti, and K. K. Szabo, Phys. Lett. B 751, 559 (2015).

[8] S. Borsanyi, Z. Fodor, J. N. Guenther, S. K. Katz, K. K. Szabo, A. Pasztor, I. Portillo, and C. Ratti, J. High Energy Phys. 10 (2018) 205.

[9] M. A. Stephanov, Phys. Rev. D 73, 094508 (2006).

[10] G. A. Almsi, B. Friman, K. Morita, and K. Redlich, Phys. Lett. B 793, 19 (2019).

[11] J. Engels and F. Karsch, Phys. Rev. D 85, 094506 (2012).

[12] M. E. Fisher, Phys. Rev. Lett. 40, 1610 (1978).

[13] A. Connelly, G. Johnson, F. Rennecke, and V. Skokov, Phys. Rev. Lett. 125, 191602 (2020).

[14] One cannot completely rule out that QCD demonstrates a weak fluctuation-induced first-order phase transition in the chiral limit for two massless quarks. Current lattice QCD calculations with staggered quarks strongly suggest that the transition is of the second order. We caution the reader that the staggered fermion lattice calculations are known to break down in the chiral limit at finite lattice spacings. The conclusion on the second order chiral phase transition is also consistent with Refs. [15-18].
[15] F. Burger, E.-M. Ilgenfritz, M. Kirchner, M. P. Lombardo, M. Mller-Preussker, O. Philipsen, C. Urbach, and L. Zeidlewicz (tmfT), Phys. Rev. D 87, 074508 (2013).

[16] T. Umeda, S. Ejiri, R. Iwami, K. Kanaya, H. Ohno, A. Uji, N. Wakabayashi, and S. Yoshida, Proc. Sci., LATTICE2016 (2017) 376.

[17] F. Cuteri, O. Philipsen, and A. Sciarra, Proc. Sci., LATTICE2018 (2018) 170.

[18] G. Endrodi and L. Gonglach, Proc. Sci., LATTICE2018 (2018) 172.

[19] H. T. Ding et al., Phys. Rev. Lett. 123, 062002 (2019).

[20] H. T. Ding, P. Hegde, F. Karsch, A. Lahiri, S. T. Li, S. Mukherjee, and P. Petreczky, Nucl. Phys. A982, 211 (2019).

[21] S. Ejiri, F. Karsch, E. Laermann, C. Miao, S. Mukherjee, P. Petreczky, C. Schmidt, W. Soeldner, and W. Unger, Phys. Rev. D 80, 094505 (2009).

[22] O. Kaczmarek, F. Karsch, E. Laermann, C. Miao, S. Mukherjee, P. Petreczky, C. Schmidt, W. Soeldner, and W. Unger, Phys. Rev. D 83, 014504 (2011).

[23] This is the so-called magnetic equation of state for threedimensional $\mathrm{O}(4)(\mathrm{Z}(2))$ universality class if $\mathrm{QCD}$ has a second (first) order phase transition in the massless limit for $\mathrm{u} / \mathrm{d}$-quarks. The mapping (2) was established for the second order phase transition in the chiral limit. Our analysis however can be extended to a first order phase transition case, if lattice QCD provides nonuniversal input on mapping parameters.

[24] D. Amit and V. Martin-Mayor, Field Theory, the Renormalization Group, and Critical Phenomena: Graphs to 
Computers Third Edition (World Scientific, Singapore, 2005).

[25] S.-T. Li and H.-T. Ding, Proc. Sci., LATTICE2016 (2017) 372.

[26] We can definitively state that the so-called thermal singularities originating from zeroes of the inverse Fermi-Dirac function will not modify our analysis as they lead to the radius of convergence $R / T<\pi$.

[27] It comes with its complex conjugate pair; this is implicitly assumed in the remaining text.

[28] F. Gliozzi and A. Rago, J. High Energy Phys. 10 (2014) 042 .

[29] C. Itzykson, R. B. Pearson, and J. B. Zuber, Nucl. Phys. B220, 415 (1983).

[30] E. Brézin and D. J. Wallace, Phys. Rev. B 7, 1967 (1973).
[31] G. A. Almasi, W. Tarnowski, B. Friman, and K. Redlich, Phys. Rev. D 95, 014007 (2017).

[32] J. Engels, L. Fromme, and M. Seniuch, Nucl. Phys. B675, 533 (2003).

[33] We thank Anirban Lahiri for providing us this input on behalf of the HotQCD collaboration. Recent results of the HotQCD collaboration on the value of $z_{0}$ can also be found in Ref. [34].

[34] D. A. Clarke, O. Kaczmarek, F. Karsch, A. Lahiri, and M. Sarkar, Phys. Rev. D 103, L011501 (2021).

[35] I. Balog, H. Chaté, B. Delamotte, M. Marohnic, and N. Wschebor, Phys. Rev. Lett. 123, 240604 (2019).

[36] S. Borsanyi, Z. Fodor, J. N. Guenther, S. K. Katz, A. Pasztor, I. Portillo, C. Ratti, and K. K. Szab, Nucl. Phys. A982, 223 (2019). 